This section features a recent systematic review that is indexed on PEDro, the Physiotherapy Evidence Database (http://www.pedro org.au). PEDro is a free, web-based database of evidence relevant to physiotherapy.

\section{Interventions to increase physical activity among older adults (PEDro synthesis)}

- Chase JA. Interventions to increase physical activity among older adults: a meta-analysis. Gerontologist 2015;55:706-18.

\section{BACKGROUND}

Chronic illness are responsible for $84 \%$ of all healthcare expenditure and are highly prevalent among the older population. ${ }^{1-3}$ Physical activity (PA) has beneficial effects on cardiovascular risk factors, physical function, pain and depressive symptoms. $^{4-7}$ There is an urgent need to understand more about how to increase PA levels in older adults.

\section{AIM}

This study aimed to investigate the effect of interventions designed to increase PA behaviour among older adults.

\section{SEARCHES AND INCLUSION CRITERIA}

The databases searched were: PubMed, MEDLINE, CINAHL, SPORTDiscus, PsychInfo, Google Scholar and Dissertation Abstracts International. The search strategy included the following combination of terms: 'exercise', 'physical activity', 'physical exertion', 'physical endurance', 'leisure activity', 'recreation', 'physical fitness' and 'physical education and training'. Studies published between 1960 and 2013, investigating PA interventions among community-dwelling older adults (aged 65 and older or with a sample mean age of 70 ), were considered eligible.

\section{INTERVENTION}

Two-arm and single-arm studies investigating any intervention designed to increase PA behaviour among older adults, such as cognitive and behavioural strategies, motivational-type interventions, theory-driven interventions and problem-solving techniques were included.

\section{MAIN OUTCOMES}

Primary outcomes included self-reported (eg, questionnaires, activity logs) and objective measures (eg, accelerometer) of PA behaviour.

\section{STATISTICAL METHODS}

The meta-analysis included two-arm studies using post-treatment scores (final values). Comprehensive Meta-Analysis Software was used to calculate standardised mean differences (SMDs) and 95\% CIs using random-effects models. The magnitude of the SMDs were interpreted as: $\leq 0.20$ represents a small effect, $=0.50$ a medium effect or $\geq 0.80$ a large effect. Moderator analyses were used to determine the impact of sample, study and intervention characteristics on the overall SMD.

\section{RESULTS}

A total of 53 two-arm studies were included in the main analysis. These studies investigated behavioural type interventions using strategies such as self-monitoring, goal setting and prompting; and/or cognitive-based interventions, including barriers management strategies, problem-solving techniques, education and counselling. More than half of these studies $(n=24)$ offered supervised exercise sessions to participants.

Overall, the results showed a small effect favouring PA interventions over control interventions with an SMD for the main analysis of 0.18 (95\% CI 0.10 to 0.26$)$. Significantly heterogeneity $\left(\mathrm{Q}=122.9, \mathrm{p}<0.001\right.$ and $\left.\mathrm{I}^{2}=57.7 \%\right)$ was found across included studies. Funnel plot inspection showed little evidence of publication bias.

Moderator analyses suggested that: (1) the effect of PA interventions was higher among healthier participants $(\mathrm{SMD}=0.30)$ than in those with history of chronic illness $(\mathrm{SMD}=0.11)$ ( $\mathrm{p}$ for comparison $=0.03)$; (2) interventions delivered through audiovisual media $(\mathrm{SMD}=0.48)$ were more effective than those that did not use this delivery method $(\mathrm{SMD}=0.14)$ ( $\mathrm{p}$ for comparison $=0.01) ;(3)$ interventions delivered via mailed materials $(S M D=0.34)$ were more effective than those without this delivery method $(\mathrm{SMD}=0.14)(\mathrm{p}$ for comparison $=0.03$ ); (4) interventions including behavioural and cognitive strategies were more effective $(\mathrm{SMD}=0.23)$ than interventions using only one strategy $(\mathrm{SMD}=0.02)$ ( $\mathrm{p}$ for comparison=0.03); (5) the effect of studies using theory-driven interventions $(\mathrm{SMD}=0.28)$ was higher than studies without a stated theoretical basis $(\mathrm{SMD}=0.05)$ ( $\mathrm{p}$ for comparison $<0.01$ ); and $(6)$ studies including motivational-type interventions were more effective $(\mathrm{SMD}=0.20)$ than studies that did not use this type of interventions $(\mathrm{SMD}=-0.15)$ ( $\mathrm{p}$ for comparison $=0.02)$.

\section{CONSIDERATIONS/LIMITATIONS}

The results of the main meta-analysis revealed a significant small effect favouring PA interventions over control. However, the high heterogeneity $\left(\mathrm{I}^{2}=57.7 \%\right)$ associated with the pooled treatment suggests that the effects reported by individual trials are variable and interpretation of the pooled effect should be made with caution. As the authors included non-randomised trials, it is possible that the effect reported is overestimated. In addition, there was no attempt to assess the risk of bias among included studies and to assess the overall quality of evidence.

\section{CLINICAL IMPLICATIONS}

Interventions specifically designed to improve PA behaviour among older adults appear to be effective, but some caution is required, given the limitations of this review. Future high-quality studies are needed, and further reviews in this area should try to incorporate current recommended methods to assess the overall quality of evidence to provide robust evidence regarding the effect of interventions designed to promote PA among older adults.

\section{Priscila K Morelhao, Crystian B Oliveira, Marcia R Franco}

Departamento de Fisioterapia, Faculdade de Ciências e Tecnologia, UNESP_U Univ Estadual Paulista, Presidente Prudente, São Paulo, Brazil

Correspondence to Marcia R Franco, Rua Roberto Simonsen, 305, FCT/UNESP, Campus de Presidente Prudente, Bairro Jardim das Rosas, Presidente Prudente, CEP: 19060-900, São Paulo, Brazil; marciarfranco@gmail.com

Contributors PKM, CBO and MRF selected the systematic review, interpreted the data and wrote the manuscript.

Competing interests None declared. 
Provenance and peer review Not commissioned; externally peer reviewed.

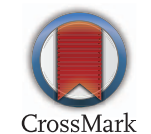

To cite Morelhao PK, Oliveira CB, Franco MR. Br J Sports Med 2017;51:1375-1376.

Accepted 28 September 2016

Published Online First 20 October 2016

Br J Sports Med 2017;51:1375-1376. doi:10.1136/bjsports-2016-096859

\section{REFERENCES}

1 Administration on Aging. A profile of older Americans: 2012. Administration for Community Living, US Department of Health and Human Services. http://www.aoa. gov/Aging_Statistics/Profile/2012/docs/2012profile.pdf (accessed 3 Jun 2016).
2 Anderson, G. Chronic care: making the case for ongoing care. Robert Wood Johnson Foundation, Baltimore: US Johns Hopkins University. http://www.rwjf.org/content/ dam/farm/reports/reports/2010/rwjf54583 (accessed 3 Jun 2016).

3 Centers for Medicare and Medicaid Services. Chronic Conditions among Medicare Beneficiaries, Chartbook, 2012 Edition, US Baltimore, 2012. https://www.cms.gov/ research-statistics-data-and-systems/statistics-trends-and-reports/chronic-conditions/ downloads/2012chartbook.pdf (accessed 3 Jun 2016).

4 Chao SF. Changes in leisure activities and dimensions of depressive symptoms in later life: a 12-year follow-up. Gerontologist 2016;56:397-407.

5 Chodzko-Zajko WJ, Proctor DN, Fiatarone Singh MA, et al., American College of Sports Medicine. American College of Sports Medicine position stand. Exercise and physical activity for older adults. Med Sci Sports Exerc 2009;41:1510-30.

6 Conn VS, Hafdahl AR, Brown SA, et al. Meta-analysis of patient education interventions to increase physical activity among chronically ill adults. Patient Educ Couns 2008;70:157-72.

7 Hughes SL, Seymour RB, Campbell R, et al. Impact of the fit and strong intervention on older adults with osteoarthritis. Gerontologist 2004;44:217-28. 